\title{
Revascularización endovascular compleja multinivel y multivaso a través de un bypass fémoro-poplíteo ocluído en paciente con isquemia crónica con amenaza para las extremidades inferiores
}

\author{
Complex multilevel and multivessel endovascular revascularization through an occluded \\ femoral-popliteal bypass in a patient with chronic limb threatening ischemia
}

Revascularização endovascular complexa multinivel e multivaso através de um bypass fêmoro-poplíteo ocluído em paciente com isquemia crônica ameaçadora de membros inferiores

Marcel Voos Budal Arins (D), Antenor Alvarez ${ }^{1}$

\begin{abstract}
Resumen
La isquemia crónica con amenaza para las extremidades inferiores (ICAEI) representa el estadio final de la enfermedad arterial periférica, un problema de prevalencia creciente que conlleva el aumento de los costos de salud en todo el mundo. La ICAEl es una enfermedad con elevada morbilidad, generando mortalidad significativa, pérdida de miembros, dolor y disminución de la calidad de vida. La principal causa de amputaciones no-traumáticas de miembros inferiores está relacionada a la diabetes y a la ICAEI. Entre un 2\% y 3\% de los pacientes con enfermedad arterial periférica se presentan con un caso grave de ICAEI, condición que se correlaciona con enfermedad arterial multinivel y multiarterial, calcificación y oclusiones totales crónicas. Se describieron varias estrategias técnicas para cruzar con éxito largas oclusiones en segmentos arteriales. Se puede realizar la recanalización utilizando técnicas endoluminales, subintimales y retrógradas. Relatamos un caso de revascularización endovascular compleja multinivel y multiarterial a través de un bypass fémoro-poplíteo en una paciente con ICAEI.
\end{abstract}

Palabras clave: isquemia crónica con amenaza para las extremidades inferiores; endovascular; angioplastia subintimal; acceso retrógrado.

\begin{abstract}
Chronic limb-threatening ischemia (CLTI) represents the end stage of peripheral artery disease, a problem of growing prevalence and increased health care costs around the globe. CLTI is a highly morbid disease, incurring significant mortality, limb loss, pain, and diminished health-related quality of life. The major cause of non-traumatic lower extremity amputation are related to diabetes and CLTI. Between 2\% to 3\% of patients with peripheral artery disease present with a severe case of CLTI, a condition that is correlated with multilevel and multivessel arterial disease, calcification, and chronic total occlusions. Multiple technical strategies to successfully cross long occlusions in arterial segments have been described. Recanalization can be performed using endoluminal, subintimal, and retrograde techniques. We report a case of complex multilevel and multivessel endovascular revascularization through an occluded femoropopliteal bypass in a patient with CLTI.
\end{abstract}

Keywords: chronic limb-threatening ischemia; endovascular; subintimal angioplasty; retrograde approach.

\section{Resumo}

A isquemia crônica ameaçadora dos membros inferiores (ICAMI) representa o estágio final da doença arterial periférica, um problema de prevalência crescente que aumenta os custos de saúde em todo o mundo. A ICAMI é uma doença altamente mórbida, causando mortalidade significativa, perda de membros, dor e diminuição da qualidade de vida. A principal causa de amputações não traumáticas de membros inferiores está relacionada com diabetes e ICAMI.

\footnotetext{
${ }^{1}$ Centro de Cardiologia Intervencionista y Terapéutica Endovascular Periférica, Hemodinamia Integral, Santiago del Estero, Argentina. Fuente de financiamiento: Ninguna. 
Entre 2\% a 3\% dos pacientes com doença arterial periférica apresentam casos graves de ICAMI, condição que está correlacionada com doença arterial multinível e multiarterial, calcificação e de oclusões totais crônicas. Foram descritas várias estratégias técnicas para cruzar com sucesso longas oclusões em segmentos arteriais. A recanalização pode ser realizada usando técnicas endoluminais, subintimais e retrógradas. Relatamos um caso de revascularização endovascular complexa multinível e multiarterial através de um bypass fêmoro-poplíteo ocluído em uma paciente com ICAMI.

Palavras-chave: isquemia crônica ameaçadora de membros inferiores; endovascular; angioplastia subintimal; acesso retrógrado.

Cómo citar: Voos Budal Arins M, Alvarez A. Revascularización endovascular compleja multinivel y multivaso a través de un bypass fémoro-poplíteo ocluído en paciente con isquemia crónica con amenaza para las extremidades inferiores. J Vasc Bras. 2022;21:e20210057. https://doi.org/10.1590/1677-5449.210057

\section{INTRODUCCIÓN}

La isquemia crónica con amenaza para las extremidades inferiores (ICAEI) representa el estadio final de la enfermedad arterial periférica (EAP), un problema de prevalencia creciente que conlleva grandes costos de atención médica en todo el mundo. La ICAEI es una enfermedad con elevada morbimortalidad, generando amputaciones, dolor y disminución de la calidad de vida ${ }^{1}$.

La principal causa de amputaciones no traumáticas está relacionada a la diabetes mellitus y a la ICAEI. Entre un 2 a $3 \%$ de los pacientes con EAP se presentan con un caso grave de ICAEI, condición que se correlaciona con la enfermedad arterial multinivel y multivaso, calcificación y oclusiones totales crónicas ${ }^{2}$.

La rápida evolución tecnológica en imágenes y dispositivos ofrece nuevas oportunidades para mejorar el tratamiento y el abordaje con técnicas endoluminales, subintimales o por vía retrógrada en estos pacientes con alto riesgo de amputación ${ }^{3}$.

El protocolo fue aprobado por el Comité de Ética de la institución (Hemodinamia Integral).

\section{Parte I - Situación clínica}

Se trata de una paciente femenina de 78 años de edad con antecedentes de hipertensión arterial, diabetes mellitus, dislipemia, extabaquismo severo, enfermedad pulmonar obstructiva crónica (EPOC) y bypass fémoro-poplíteo en miembro inferior derecho hace 7 años por claudicación intermitente grave (Rutherford 3).

La paciente consulta por ICAEI (Rutherford 5), con dolor de reposo y una úlcera en el talón derecho [classificación Wound, Ischemia and foot Infection (WIfI) 231]. La angiografía del miembro inferior derecho reveló la oclusión de las arterias iliaca externa, femoral común, femoral superficial, tibial anterior, oclusión del segmento P1 de la arteria poplítea y oclusión del bypass fémoro-poplíteo. El tronco tibioperoneo, la arteria peronea y la arteria tibial posterior se encontraban permeables (Figura 1).
La paciente fue descartada para cirugía abierta por su antecedente de EPOC.

\section{Parte II - Qué se hizo}

Se hizo punción de la arteria femoral común izquierda y colocación de introductor Destination $6 \mathrm{Fr}$ de $45 \mathrm{~cm}$ en la arteria iliaca primitiva derecha por la técnica de up and over.

Hubo un intento fallido de revascularización anterógrada con un catéter de soporte vertebral $4 \mathrm{Fr}$ y guías 0,018 " (V-18) e hidrofílicas 0,035 ".

Se realizó abordaje por la cara anterolateral de la pierna derecha y punción guiada por roadmapping del segmento P3 de la arteria poplítea con aguja de $21 \mathrm{G}$ (Figura 2A).

Con un catéter de soporte Rubicon 18 y una guía V-18 con técnica sin introductor (sheathless), se logró progresar por vía retrógrada la guía hasta la arteria iliaca externa, a través del bypass fémoro-poplíteo (Figura 2B). La guía se encontraba en posición subintimal y no fue posible reentrar por vía retrógrada. Luego, por vía anterógrada, se realizó técnica CART con un balón de 5,0 $\mathrm{mm}$ de diámetro (Figura 2C) y la técnica rendezvous (Figura 2D), externalizándose la guía retrógrada por el acceso femoral izquierdo.

Se realizó pre-dilatación de arteria poplítea con balón de $6,0 \mathrm{~mm}$ de diámetro por $100 \mathrm{~mm}$ de longitud y de arteria femoral común e iliaca externa derecha con balón de 7,0 $\mathrm{mm}$ de diámetro por $150 \mathrm{~mm}$ de longitud.

Se implantó un stent autoexpandible de nitinol Supera de 5,5 $\mathrm{mm}$ de diámetro por $200 \mathrm{~mm}$ de longitud en arteria poplítea y porciones distal y media del bypass. Además, hubo la implantación de de stent autoexpandible de nitinol Supera de $6,5 \mathrm{~mm}$ de diámetro por $180 \mathrm{~mm}$ de longitud en porción proximal del bypass y la arteria femoral común; y también un stent autoexpandible de nitinol Epic de $8,0 \mathrm{~mm}$ de diámetro por $120 \mathrm{~mm}$ de longitud en las arterias iliaca externa e iliaca primitiva. Por fin, se logró endohemostasia del sitio de punción retrógrada con un balón de 4,0 mm de diámetro. 


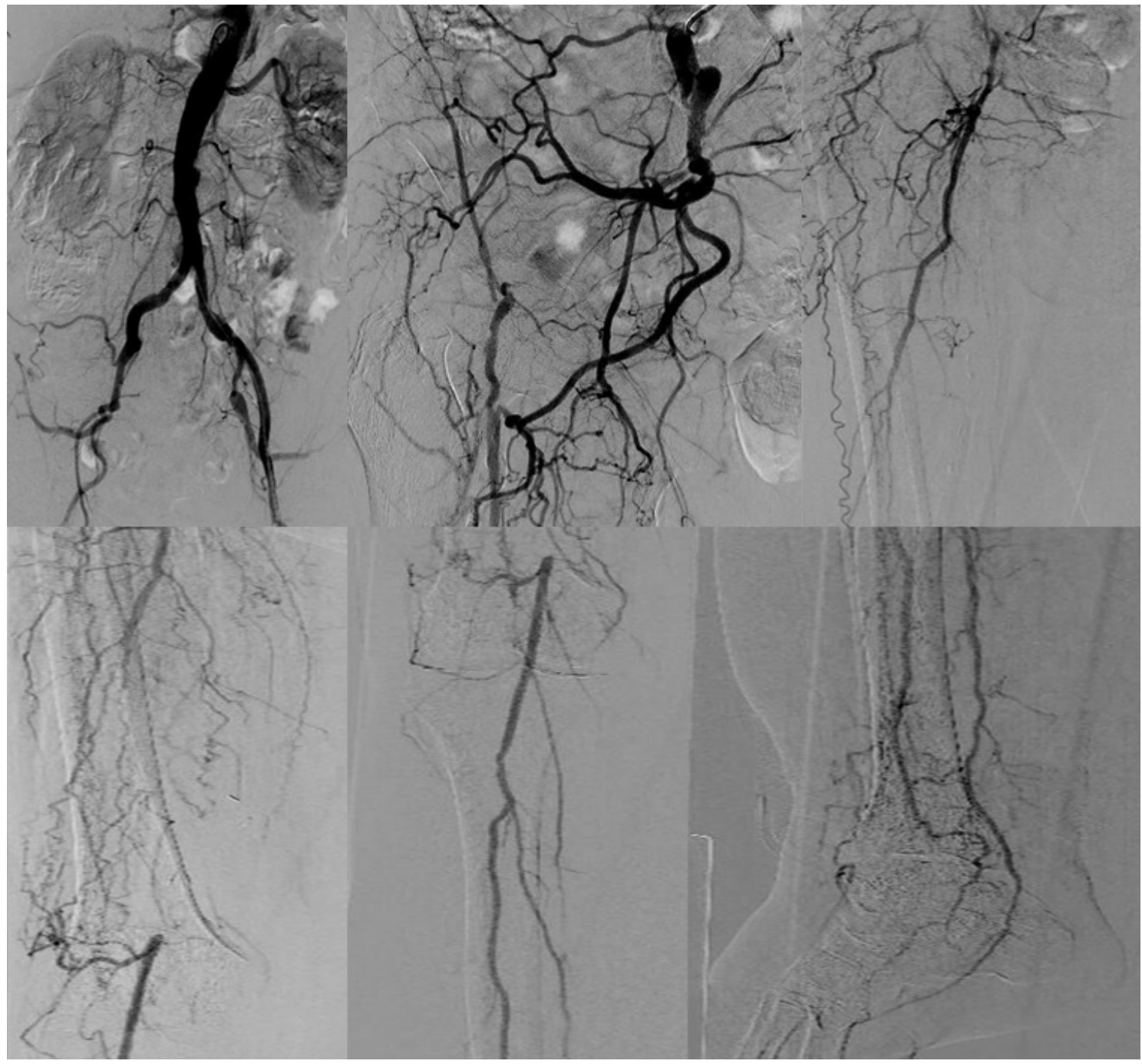

Figura 1. Se observa extensa enfermedad arterial multinivel y multivaso, con presencia de calcificación y de oclusiones totales crónicas.

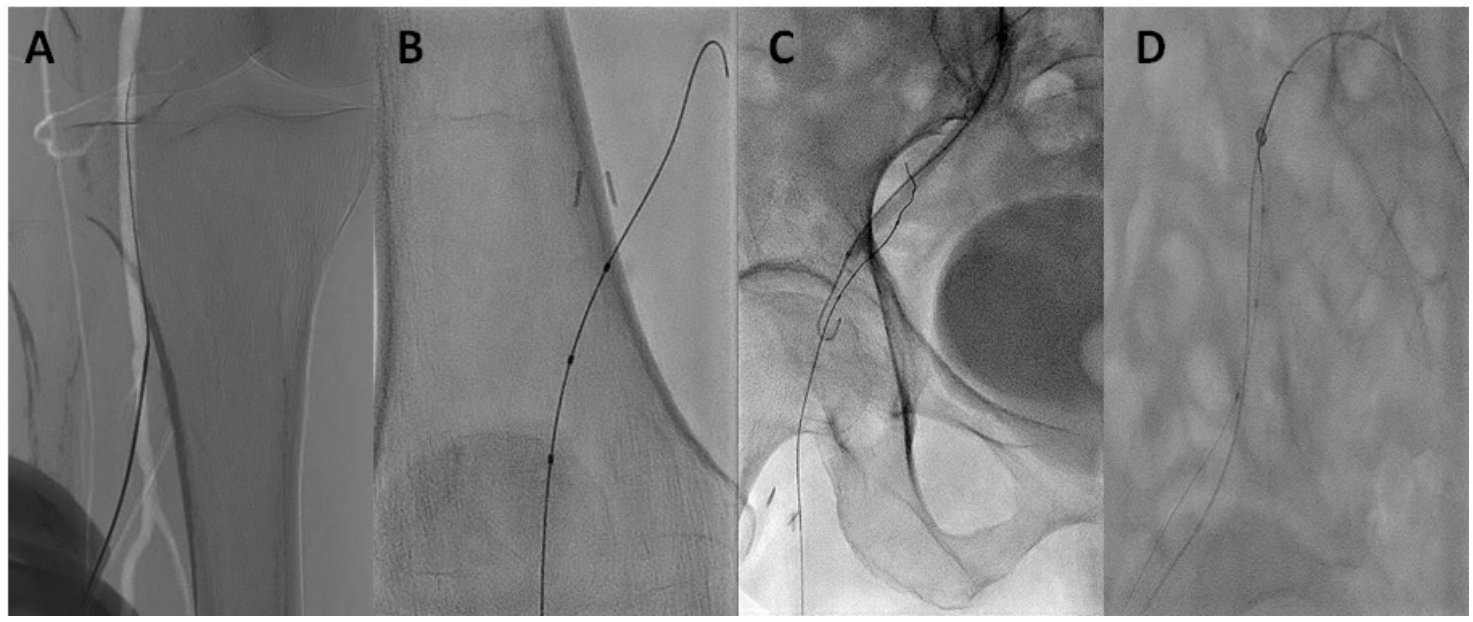

Figura 2. (A) Punción guiada por roadmapping del segmento P3 de la arteria poplítea; (B) Catéter Rubicon 18 y guía V-18 con técnica sin introductor (sheathless) ingresando al bypass fémoro-poplíteo por vía retrógrada; (C) Técnica CART; (D) Técnica rendezvous. 
El control angiográfico reveló buen resultado en los segmentos tratados, embolización a nivel de la porción distal de la arteria peroneal y buen flujo anterógrado en la arteria tibial posterior. Se observó buen flujo en la rama calcánea media de la arteria tibial posterior que irrigaba el angiosoma correspondiente al sitio de la úlcera y buen flujo en ambas arterias plantares (Figura 3).
La paciente fue seguida de forma ambulatoria con examen físico y control con ecografía Doppler a los 30, 60 y 90 días post-angioplastia. El examen físico mostró curación completa de la úlcera en el talón. Control con ecografía Doppler no reveló señales de fractura de los stents, demostrando flujo laminar, sin turbulencias ni señales de hiperplasia neointimal (Figura 4).

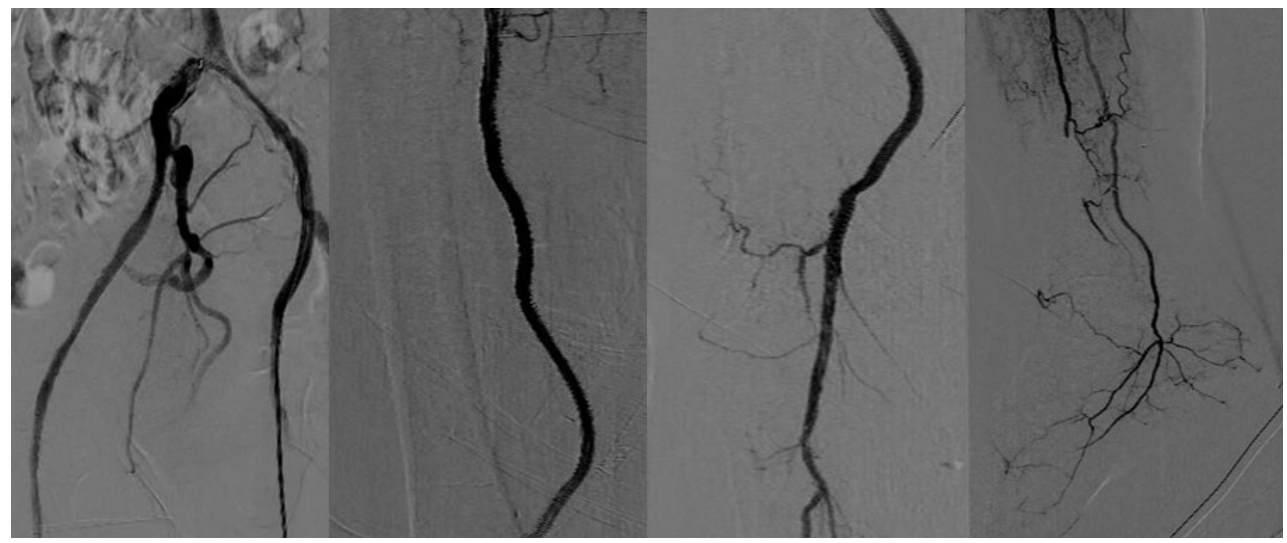

Figura 3. Control angiográfico final con buen flujo directo hacia el pié.

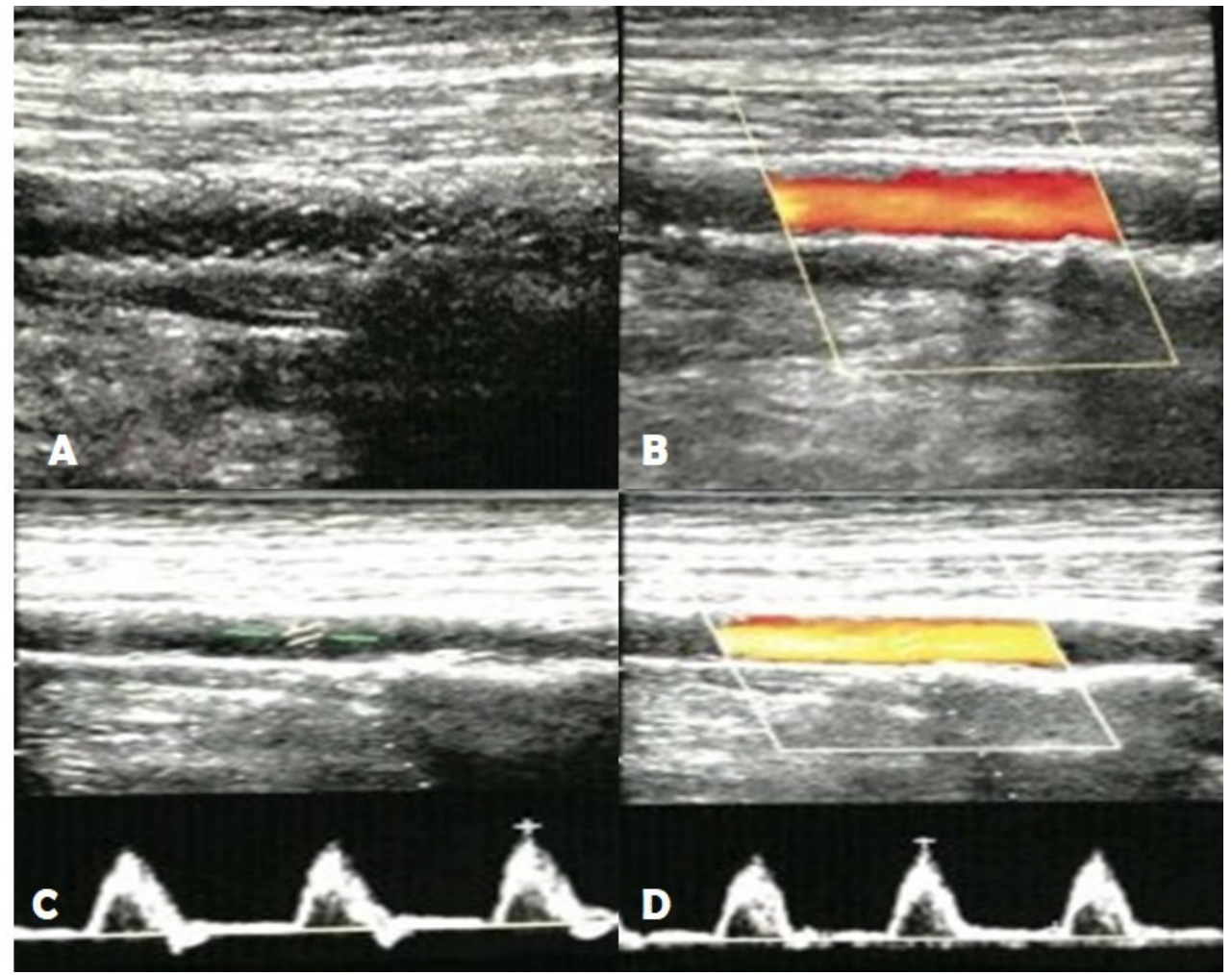

Figura 4. Ecografía Doppler a los 90 días post-angioplastia. (A) Modo B: Stent en arteria poplítea sin señales de fractura; (B) Doppler: Stent con flujo laminar, sin turbulencias ni señales de hiperplasia neointimal; (C) Modo B: Arteria poplítea distal sin placas aterosclerótica significativas; (D) Doppler arteria poplítea distal: Curva trifásica con velocidad de pico sistólica de $74 \mathrm{~cm} / \mathrm{s}$. 
El consentimiento informado es suficiente para la confección del manuscrito.

\section{DISCUSIÓN}

La ICAEI es el resultado de la enfermedad arterial oclusiva multinivel ${ }^{1}$. Los pacientes con ICAEI tienen múltiples comorbilidades, y la elección del tratamiento inicial requiere un enfoque terapéutico personalizado que equilibre la clínica del paciente con las limitaciones técnicas y anatómicas ${ }^{4}$.

La característica mínimamente invasiva de la terapéutica endovascular permite que sea aplicada a gran parte de la población, especialmente a los que tienen elevada comorbilidad ${ }^{4}$, como es el caso de nuestra paciente.

Durante el tratamiento endovascular de la ICAEI, es fundamental adaptar la estrategia técnica del procedimiento a las indicaciones clínicas y al riesgo de amputación ${ }^{5}$.

Por tratarse de una paciente descartada para cirugía abierta por elevada comorbilidad, con alto riesgo de amputación y con una anatomía vascular desafiante y compleja, se optó por una estrategia endovascular agresiva con accesos anterógrado, retrógrado, técnicas endoluminales, subintimales, de reentrada (CART) y de reconexión con el acceso anterógrado (rendezvous).

Estas técnicas, asociadas a la buena calidad de imagen y a la utilización de dispositivos específicos, proporcionan buenos resultados clínicos en términos de salvataje del miembro y supervivencia libre de amputación.

En conclusión, la decisión de utilizar técnicas endovasculares complejas debe estar guiada por el estado clínico del paciente y no sólo por la anatomía vascular.

Contamos con muchas técnicas para abordar anatomías complejas $\mathrm{y}$, al combinarlas de forma adecuada, siguiendo las necesidades clínicas, podemos lograr buenos resultados, evitando amputaciones y disminuyendo la morbimortalidad de los pacientes.

\section{REFERENCIAS}

1. Conte MS, Bradbury AW, Kolh P, et al. Global vascular guidelines on the management of chronic limb-threatening ischemia. J Vasc Surg. 2019;69(6S):3S-125S.e40. http://dx.doi.org/10.1016/j. jvs.2019.02.016. PMid:31159978

2. Faglia E. Characteristics of peripheral arterial disease and its relevance to the diabetic population. Int J Low Extrem Wounds. 2011;10(3):152-66. http://dx.doi.org/10.1177/1534734611417352. PMid:21856972.

3. Ferraresi R, Palena $L M$, Mauri G, Manzi M. Tips and tricks for a correct "endo approach... J Cardiovasc Surg. 2013;54(6):685-711. PMid:24126507.

4. Lin JH, Brunson A, Romano PS, Mell MW, Humphries MD. Endovascular-first treatment is associated with improved amputation-free survival in patients with critical limb ischemia. Circ Cardiovasc Qual Outcomes. 2019;12(8):e005273. http://dx.doi. org/10.1161/CIRCOUTCOMES.118.005273. PMid:31357888.

5. Palena LM. An extreme approach to CLI revascularization. Endovascular Today. 2016;15(5):68-72.

Correspondencia Marcel Voos Budal Arins Centro de Cardiologia Intervencionista y Terapéutica Endovascular Periférica, Hemodinamia Integral 9 de Julio, 580 4200 - Santiago del Estero, Argentina Tel.: +5491144082595 E-mail:marcelvoos@gmail.com

Informaciones sobre los autores MVBA y AA - Médicos cardiólogos intervencionistas graduados, Universidad de Buenos Aires (UBA).

Contribución de los autores Concepción y diseño del estudio: MVBA, AA Análisis e interpretación de los datos: MVBA, AA Recolección de datos: MVBA, AA Redacción del artículo: MVBA, AA Revisión crítica del texto: MVBA, AA Aprobación final del artículo*: MVBA, AA Análisis estadístico: MVBA, AA Responsabilidad general del estudio: MVBA, AA

*Todos los autores leyeron y aprobaron la versión final sometida al J Vasc Bras. 